\title{
Joint Optimization of Power Allocation and Load Balancing for Hybrid VLC/RF Networks
}

\author{
Mohanad Obeed, Student Member, IEEE, Anas M. Salhab, Senior Member, IEEE, Salam A. Zummo, Senior \\ Member, IEEE, and Mohamed-Slim Alouini, Fellow, IEEE
}

\begin{abstract}
In this paper, we propose and study a new joint load balancing (LB) and power allocation (PA) scheme for a hybrid visible light communication (VLC) and radio frequency (RF) system consisting of one RF access point (AP) and multiple VLC APs. An iterative algorithm is proposed to distribute the users on the APs and distribute the powers of the APs on their users. In PA subproblem, an optimization problem is formulated to allocate the power of each AP to the connected users for the total achievable data rate maximization. In this subproblem, we propose a new efficient algorithm that finds the optimal dual variables after formulating them in terms of each other. This new algorithm provides faster convergence and better performance than the traditional subgradient method. In addition, it does not depend on the step size and the initial values of the variables, which we look for, as the subgradient does. Then, we start with the user of the minimum data rate seeking for other AP that offers a higher data rate for that user. Users with lower data rates continue re-connecting from AP to other to balance the load only if this travel increases the summation of the achievable data rates and enhances the system fairness. Two approaches are proposed to have the joint PA and LB performed: a main approach that considers the exact interference information for all users, and a suboptimal approach that aims to decrease the complexity of the first approach by considering only the approximate interference information of users. The numerical results demonstrate that the proposed algorithms improve the system capacity and system fairness with fast convergence ${ }^{1}$.
\end{abstract}

Index Terms-Hybrid visible light communication and radio frequency (VLC/RF), power allocation, load balancing.

\section{INTRODUCTION}

$\mathbf{T}$ HE growing demand for high data rate and licencefree spectrum applications has stimulated recently many researchers to investigate the visible light communication (VLC) as a promising technique for indoor communication. As known, the radio frequency (RF) communications are becoming more restricted because of the limited spectrum resources in wireless networks. As a solution, the VLC has been introduced in indoor environment to overcome the RF limitations and provide better services to the users.

The VLC is a communication system that uses light emitting diode (LED), which is highly energy efficient, as a transmitter to emit both the light and information signals to the users. The receiver must be equipped with a photo detector device that converts the light signal to an electrical signal. Hence, the data is transmitted using an intensity modulation (IM) scheme at the transmitter and received using a direct detection (DD) scheme at the receiver (IM/DD). In addition for being license-free and

\footnotetext{
${ }^{1}$ This work is an extended version of our paper which has been recently accepted and presented in IEEE GLOBECOM Conference 2017 [1].
}

secure systems, the VLCs are known for their ability to reject RF interference and their high bandwidth potential [2]. On the other hand, the VLC suffers from the blockages. Unlike the RF network, the VLC works properly only when the Line of site (LoS) component between the transmitter and receiver is available. Therefore, supplementing the VLC network with a RF AP increases the network coverage and improves the total system capacity [3].

Authors of [4] considered the cooperation between two VLC APs to increase the rate region by controlling the transmission power. In [5], the authors studied the advantages of combining the RF and VLC APs with showing how this combining enhances the system throughput and decreases the delay. In [6], the authors showed that the users are distributed dynamically on both the VLC and RF networks based on the user channel condition. Then, the users with lower achievable rates are migrated to the RF system to increase their data rates. To decrease the number of handovers, [7] proposed a dynamic load balancing (LB) scheme where the quasi-static users are connected to VLC APs, while the unfixed users are connected to RF AP. To study the joint load balancing and time resource allocation, [8] proposed a bidirectional allocation game where each user is initially serviced from the AP that provides the highest signal-to-noise ratio (SNR) with time resource allocation. The unsatisfied users are then reconnected to an adjacent AP only in case that it can offer a better service. The procedures of resource allocation and load balancing are repeated, until no more handover occurs. In [9], power and bandwidth allocation was investigated for energyefficient hybrid VLC/RF system consisting of only one VLC AP and one RF AP. Since all the users (either served by RF AP or VLC AP) do not suffer from interference, the problem formulated in [9] is easy to tackle. Authors of [10] studied how the APs should cooperate to mitigate the interference with balancing the load, but the power allocation problem has not been considered. While authors of [11] and [12] extended the work of [10] by studying the cell formation with allocating the power for only VLC network. However, the problems of associating the users and allocating the power have been tackled separately in [11] and [12], where they are interlinked problems and they must be tackled jointly. Up to the authors knowledge, the joint LB and power allocation (PA) for a hybrid VLC/RF network has not been presented or studied before.

In this paper, different from the above literature mentioned, we study the two problems of PA and LB in a hybrid VLC/RF network for the sake of data rate maximization and system fairness improvement. The network consists of multiple VLC 
APs and one RF AP. First, each user is connected to its closest AP. Then, each AP performs its optimization problem (allocates the power for the associated users) in order to maximize the summation of the achievable data rates per AP. After that, the users with the lower data rates start reconnecting from AP to other to balance the load only in case this transfer increases the summation of the achievable data rates. This transfer of users continues until no improvement in the summation of data rates is achieved. We prove the convergence of the proposed algorithm analytically and numerically. The inter-cell interference makes the joint PA and LB problem very difficult. Therefore, two approaches are proposed to have the joint PA and LB implemented: 1) the approach that considers the instantaneous (exact) interference information for all users, 2) and a suboptimal approach that aims to decrease the complexity of the first approach by considering only the approximate interference information of users. This work is different from the work of [8] in several aspects as follows: 1) we generalize the system to include a RF AP, 2) in the PA subproblem, the authors in [8] performed a time resource allocation problem, while we allocate the power for the users, 3) we formulate the PA problem as a mathematical optimization problem and not by using the fuzzy logic as in [8], and 4) and we propose a suboptimal approach to decrease the complexity of the procedures significantly with a negligible loss in the performance. The procedures are simplified in such a way when a user connection is transferred, only two APs perform the power allocation problem and not all the APs as in [8]. In the PA optimization subproblem, in the VLC and RF APs, we formulate the problem for maximizing the summation of the achievable users data rates under certain QoS constraints. These QoS constraints are formulated to control the tradeoff between the system capacity and system fairness. For a given interference information, the PA problem is proved to be concave but not easy to tackle. Similar PA problems were studied before such as [9], [13], [14], [15], [16], and [17]. In finding the dual variables, the authors of these references used the subgradient method, which is very sensitive to step size selection and needs a large number of iterations for convergence. Here, we derive a new efficient algorithm that finds the optimal dual variables after formulating them in terms of each other without requiring to optimize the step size or selecting the initial values carefully. This new algorithm provides faster convergence and better performance than the traditional subgradient method.

The rest of this paper is organized as follows. The system and channel models are introduced in Section II. In Section III, we present the problem formulation and proposed algorithms. Some simulation results are presented and discussed in Section IV. Finally, the paper is concluded in Section V.

\section{SySTEM AND CHANNEL MODELS}

The system under consideration consists of $N_{a p}$ VLC APs, one RF AP, and $N_{u}$ users as shown in Fig. 1. The users are distributed uniformly in the room and the APs are fixed in the ceiling of the room. Each VLC AP is equipped with multiple LEDs that use IM to transmit the light signal to the users, which receive the light by a photo detector (PD). The RF AP is assumed to cover all the room area. Also, the location of users are assumed to be unchanged during a short period of interest $T$. Thus, the VLC and RF channel-state-information (CSI) of both the VLC and RF links is considered to be constant during this period. We assume that the maximum available bandwidth at the AP $i$ is divided fairly among all the users connected to that AP.

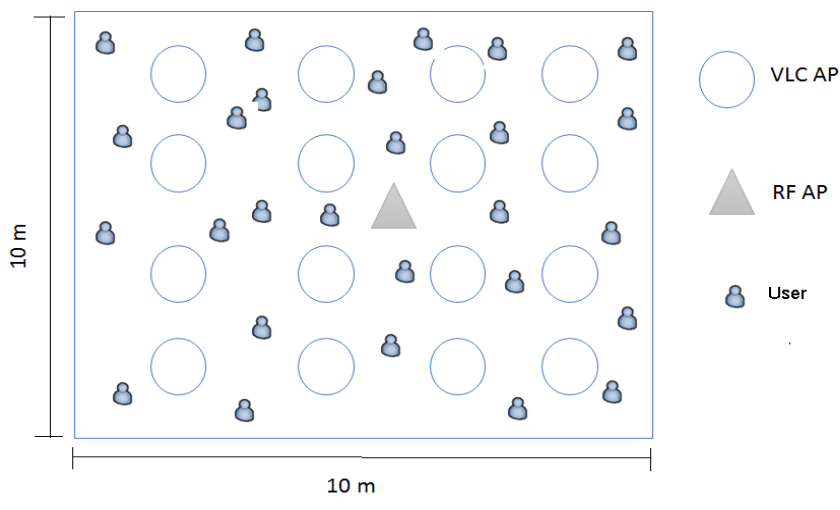

Fig. 1. System model.

\section{A. VLC Channel}

The LoS VLC channel between the $i^{\text {th }}$ LED and the $j^{t h}$ user can be modeled as follows [18]

$$
h_{j, i}^{(v)}=\frac{(m+1) A_{p}}{2 \pi d_{j, i}^{2}} \cos ^{m}(\phi) g_{o f} f(\theta) \cos (\theta),
$$

where $m$ is the Lambertian index that is given by $m=$ $-1 / \log _{2}\left(\cos \left(\theta_{1 / 2}\right)\right.$, where $\theta_{1 / 2}$ is the half intensity radiation angle, $A_{p}$ is the physical area of the receiver $\mathrm{PD}, d_{j, i}$ is the distance between the $i^{\text {th }}$ AP to the $j^{t h}$ user, $g_{o f}$ is the gain of the optical filter, $\phi$ is the angle of radiance at the AP, $\theta$ is the angle of incidence at the $\mathrm{PD}$, and $f(\theta)$ is the optical concentrator gain, which is a function of $\theta$ that is given by

$$
f(\theta)= \begin{cases}\frac{n^{2}}{\sin ^{2}(\Theta)}, & 0 \leq \theta \leq \Theta ; \\ 0, & \theta>\Theta,\end{cases}
$$

where $n$ is the refractive index and $\Theta$ is the semi-angle of the field-of-view (FoV) of PD. In a VLC network, the LED has to operate in the linear region so that the optical power at its output is a linear function of the input voltage. In VLC networks, the signal is deteriorated significantly in the nonLoS VLC transmissions that might lead to unsuccessful data transmission [8]-[10], so that we work only on LoS paths. This assumption does not affect the proposed algorithm since it does not depend on a specific channel model. In other words, whatever the channel model is, the proposed algorithm for the power allocation will give us the optimal solution as will be shown. Therefore, when the LoS path is available, $h_{j, i}^{(v)}$ is given by (1); otherwise, $h_{j, i}^{(v)}=0$. The probability of the availability of the LoS from an AP to a user is denoted by $\alpha$ and it is assumed to be uniformly distributed. It was shown in [9] that 
the average electrical power of the received signal at user $j$ from the VLC AP $i$ is calculated as follows

$$
P_{\text {elec }, j, i}=\left(\rho \sqrt{P_{j, i}^{(v)}} h_{j, i}^{(v)}\right)^{2},
$$

where $\rho$ is the optical-to-electric conversion efficiency, $P_{j, i}^{(v)}$ is the allocated power for user $j$ from the $\mathrm{AP} i$, where $P_{i, \max }^{(v)}$ is the maximum transmitted optical power of the AP $i$. The received SNR at user $j$ from the VLC AP $i$ can be expressed as

$$
\Gamma_{j, i}^{(v)}=\frac{\left(\rho \sqrt{P_{j, i}^{(v)}} h_{j, i}^{(v)}\right)^{2}}{\left(B_{i, \max }^{(v)} / N_{i}\right) N_{0}^{(v)}+\rho^{2} \sum_{l=1, l \neq i}^{N_{p}} P_{B_{j, l}}^{(v)} h_{j, l}^{(v) 2}},
$$

where $B_{i, \text { max }}^{(v)}$ is the maximum available bandwidth at the AP $i, N_{i}$ is the number of users connected to the $\mathrm{AP} i, N_{0}^{(v)}$ is the noise power spectral density, and $P_{B_{j}, l}^{(v)}$ is the interference power at user $j$ caused by the AP $l$. It is worth to note that $P_{B_{j, l}}^{(v)}$ must be calculated carefully since it represents the power allocated from the AP $l$ to the same frequency spectrum that is allocated for user $j$. For instance, if the AP $l$ is associated with 4 users, and user $j$ along with another user are connected to AP $i, P_{B_{j}, l}^{(v)}$ should be calculated as the summation of the powers of 2 users out of the 4 users that are connected to the AP $l$ and use the same spectrum frequency used by user $j$.

Here, Shannon's capacity equation is used to express the achievable data rate that represents a limit for the performance without considering specific modulation and coding schemes. This equation is employed due to its mathematical tractability since using different modulation schemes does not affect significantly the optimality of the achieved data rate [19]. Therefore, according to [9], [10], the maximum data rate that can be achieved at user $j$ from the VLC AP $i$ is given by

$$
R_{j . i}^{(v)}=\left(B_{i, \max }^{(v)} / N_{i}\right) \log _{2}\left(1+\Gamma_{j, i}^{(v)}\right) .
$$

\section{B. RF Channel}

As mentioned before, the RF cell is assumed to cover all the room area and a spectrum of a total bandwidth $B_{\max }^{(r)}$ at the RF AP is considered to be flat fading and can be partitioned into non-overlapping channels of unequal bandwidths. According to [3], the RF channel gain between user $j$ and the RF AP is expressed as

$$
h_{j}^{(r)}=\sqrt{10^{-\frac{L(d)}{10}}}\left(\sqrt{\frac{K}{K+1}} h_{d}+\sqrt{\frac{1}{K+1}} h_{s}\right),
$$

where $K=10 \mathrm{~dB}$ is the Rician factor for indoor $60 \mathrm{GHz}$ mmWave links, $h_{d}=\sqrt{0.5}(1+j)$ is the LoS fading channel, $h_{s}$ is a circular normal distributed with zero mean and unity variance and it is the fading channel of the scattered path, and $L(d)$ is the corresponding large-scale fading loss in decibels at the separation distance $d$, given by

$$
L(d)=L\left(d_{0}\right)+10 v \log _{10}\left(d / d_{0}\right)+Z,
$$

where $L\left(d_{0}\right)=68 \mathrm{~dB}$ is the reference path loss at some reference distance $d_{0}=1 \mathrm{~m}, v=1.6$ is the path loss exponent, and $Z$ is the shadowing component, which is assumed to be a zero mean Gaussian distributed random variable with a standard deviation of $1.8 \mathrm{~dB}$. The shadowing effect induced by human bodies in the proximity of the mmWave radio links is omitted [3]. The data rate achieved by the RF link between user $j$ and the RF AP can be expressed as

$$
\Gamma_{j}^{(r)}=\frac{P_{j}^{(r)}\left|h_{j}^{(r)}\right|^{2}}{\left(B_{\max }^{(r)} / N_{r}\right) N_{0}^{(r)}},
$$

where $B_{\max }^{(r)}$ is the maximum available bandwidth at the RF $\mathrm{AP}$ and $N_{r}$ is the number of users connected to the RF AP. The achievable data rate that can be achieved by user $j$ from the RF AP is given by

$$
R_{j}^{(r)}=\left(B_{\max }^{(r)} / N_{r}\right) \log _{2}\left(1+\Gamma_{j}^{(r)}\right) .
$$

\section{PRoblem Formulation}

The problem here is how to assign all the users to the APs and how to allocate the APs powers for the assigned users aiming to maximize the system capacity with keeping the fairness between the users at an acceptable level. Therefore, we start by assigning the users based on their distance from the APs, where each user selects the closest AP to it. Then, each AP performs its own power allocation problem for its associated users.

The achievable data rate of user $j$ is expressed as

$$
R_{j, i}= \begin{cases}R_{j, i}^{(v)}, & i \in \mathcal{C}_{v} ; \\ R_{j, i}^{(r)}, & i \in \mathcal{C}_{r},\end{cases}
$$

where $\mathcal{C}_{v}$ denotes the set of the VLC APs and $\mathcal{C}_{r}$ denotes the RF AP.

\section{A. Power Allocation in VLC and RF Access Points}

In this section, we aim to allocate the power for the $N_{i}$ users that are connected to the AP $i$. The objective function here is to maximize the summation of the users data rates under certain QoS constraints. These constraints are formulated to guarantee some fairness for users per APs. We formulate the optimization problem as a general form for the VLC APs or the RF AP. Hence, $\left[\begin{array}{llll}P_{j, i} & B_{j, i} & h_{j, i} & N_{0}\end{array}\right]=$ $\left[\begin{array}{llll}P_{j, i}^{(v)} & \left(B_{i, \max }^{(v)} / N_{i}\right) & \rho h_{j, i}^{(v)} & N_{0}^{(v)}\end{array}\right]$, if the AP $i$ is a VLC $\mathrm{AP}$, and if the AP $i$ is a $\mathrm{RF} \mathrm{AP}$, so $\left[\begin{array}{llll}P_{j, i} & B_{j, i} & h_{j, i} & N_{0}\end{array}\right]=$ $\left[\begin{array}{llll}P_{j}^{(r)} & \left(B_{\max }^{(r)} / N_{r}\right) & h_{j}^{(r)} & N_{0}^{(r)}\end{array}\right]$. Therefore, the optimization problem of the AP $i$ can be expressed as

$$
\begin{array}{cl}
\max _{P_{1, i}, ., P_{N_{i}, i}} & \sum_{j=1}^{N_{i}} R_{j, i} \\
\text { s.t. } & R_{j, i} \geq \gamma_{j, i}, j=1, . ., N_{i} \\
& \sum_{j=1}^{N_{i}} P_{j, i} \leq P_{i, \max }, \\
& P_{j, i} \geq 0 \quad \forall j,
\end{array}
$$

where $N_{i}$ is the number of users associated with the $\mathrm{AP} i$, and $\gamma_{j, i}$ is the minimum data rate that can be achieved at user $j$ from the AP $i$, which is given by

$$
\gamma_{j, i}=\beta \frac{B_{i, \max }}{N_{i}} \log \left(1+\frac{\left(P_{i, \max } / N_{i}\right)\left|h_{j, i}\right|^{2}}{\left(B_{i, \max } / N_{i}\right) N_{0}+X_{j, i}}\right),
$$


where $X_{j, i}$ is the interference term of user $j$ and is equal to $\rho^{2} \sum_{l=1, l \neq i}^{N_{p}} P_{B_{j, l}}^{(v)} h_{j, l}^{(v) 2}$ if the AP $i$ is VLC, and in the case of RF AP, $X_{j, i}=0$, and $\beta$ is a value in the interval $[0,1]$. If we select $\beta=1$, this means that the AP will distribute its resources fairly (regardless of users channels), where each user gains the same power $P_{i, \max } / N_{i}$ and same bandwidth $B_{i, \max } / N_{i}$. On the other hand, if $\beta$ is close to zero, this means that the objective function is released from the first constraint, which leads to increase the AP capacity.

Jain's fairness index is used to measure the AP fairness and the whole system fairness. Therefore, the fairness of the AP $i$ is given by

$$
F_{i}=\frac{\left(\sum_{j=1}^{N_{i}} R_{j, i}\right)^{2}}{N_{i} \sum_{j=1}^{N_{i}} R_{j, i}^{2}},
$$

and the fairness of the system is given by

$$
F_{s}=\frac{\left(\sum_{i=1}^{N_{a p}} \sum_{j=1}^{N_{i}} R_{j, i}\right)^{2}}{N_{a p} \sum_{i=1}^{N_{a p}} \sum_{j=1}^{N_{i}} R_{j, i}^{2}} .
$$

The Problem in (11) is not easy to tackle since in the case where the AP $i$ is a VLC AP, the interference term in the objective function makes the problem difficult. On the other hand, if the interference terms are given, the Problem in (11) becomes a concave problem and can be solved by using the Lagrangian dual problem. First, we solve this problem under the assumption that the interference terms are given then we provide an iterative algorithm that achieves the optimal power allocation. Obviously, the Constraints in (11c) and (11d) are linear functions, while the objective function and the Constraint in (11b) are well known as concave functions as shown in [9] and [14]. Therefore, the optimization Problem in (11) is a concave problem with one global optimum solution. Hence, we can use the dual problem to achieve the optimal solution, where the strong duality holds in the concave problems. The dual optimization problem of the Problem in (11) can be expressed as follows

$$
\begin{array}{r}
\zeta=-\sum_{j=1}^{N_{i}} R_{j, i}-\sum_{j=1}^{N_{i}} \mu_{j, i}\left(R_{j, i}-\gamma_{j, i}\right) \\
+v_{i}\left(\sum_{j=1}^{N_{i}} P_{j, i}-P_{i, \max }\right),
\end{array}
$$

where $\mu_{j}$ is the Lagrangian multiplier for the data rate constraint of the $j^{\text {th }}$ user and $v_{i}$ is the Lagrangian multiplier for the total power constraint.

In the following, an efficient algorithm is proposed to solve the Problem in (11) by minimizing the dual problem in (15). From the Karush-Kuhn-Tucker (KKT) conditions [20], we have

$$
\frac{\partial \zeta}{\partial P_{j, i}}=0
$$

where

$$
P_{j, i}=\frac{\left(1+\mu_{j, i}\right) B_{j, i}}{v_{i} \ln (2)}-\frac{N_{0} B_{j, i}+X_{j, i}}{\left|h_{j, i}\right|^{2}},
$$

where the variable $\mu_{j, i}$ must guarantee that the Constraint in (11b) is feasible and $v_{i}$ must guarantee that the Constraint in (11c) is feasible too. Now, these dual variables must be found to obtain $P_{j, i} \forall j$. In several papers such as [9], [13], [14], [15], [16], and [17], the authors found such dual variables by using the gradient decent method, which is an iterative algorithm that needs a large number of iterations to converge, a very careful selection of the step size, and a careful initial values selection for the dual variables. Proposing another approach that get red off these requirements (optimizing step size and the careful selection of the initial values) significantly simplifies the problem and provides a better performance. Here, we find a closed-form expression for $v_{i}$ in terms of $\mu_{j, i}$ and vise versa. Then, we solve them alternatively until they converge. First, it is trivial showing that the Constraints in (11c) must hold with equality at optimality. Otherwise, we can increase one of the power variables until the constraints hold with equality, which leads to increasing the objective function, and hence, contradicting the optimality. By substituting (17) in (11c), we have

$$
v_{i}=\frac{\sum_{j=1}^{N_{i}} B_{j, i}\left(1+\mu_{j, i}\right)}{\ln (2)\left(P_{i, \max }+\sum_{j=1}^{N_{i}} \frac{B_{j, i} N_{0}+X_{j, i}}{\left|h_{j, i}\right|^{2}}\right)} .
$$

Similarly, by substituting (17) in (11b), $\mu_{j, i}$ must be

$$
\mu_{j, i} \geq \frac{v_{i} \ln (2) P_{j, i}^{\min }}{B_{j, i}}+\frac{v_{i} \ln (2)\left(B_{j, i} N_{0}+X_{j, i}\right)}{B_{j, i}\left|h_{j, i}\right|^{2}}-1,
$$

where $P_{j, i}^{\min }$ is given by

$$
P_{j, i}^{\min }=\frac{\left(2^{\left(\gamma_{j, i} / B_{j . i}\right)}-1\right)\left(B_{j, i} N_{0}+X_{j, i}\right)}{\left|h_{j, i}\right|^{2}}, j=1, . ., N_{i},
$$

which is the minimum required power to achieve Constraints (11b). Hence, (18) and (19) depend on each other and can be solved alternatively starting from an initial value of one of them until they converge. After that, (17) is used to find the optimal power allocation. Algorithm 1 is proposed to solve the optimization problem in (11) with a given interference information. Condition 5 in Algorithm 1 examines the convergence

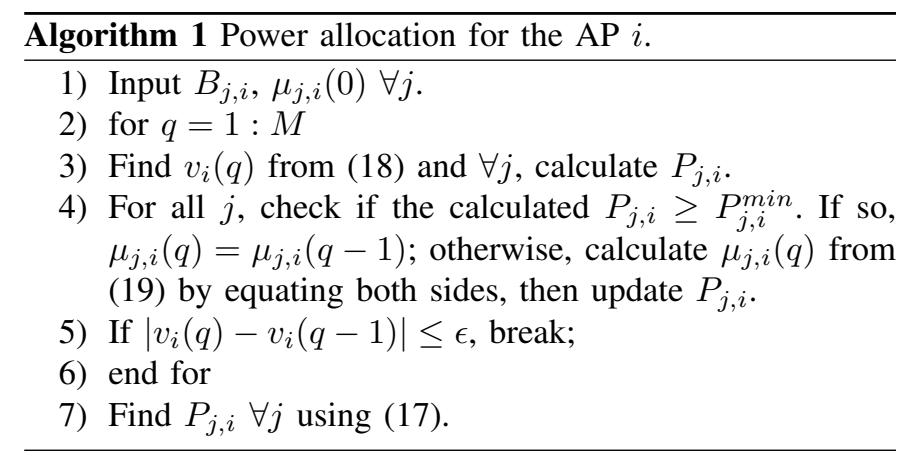

of all dual variables $v_{i}$ and $\mu_{j, i}, j=1,2, . ., N_{i}$.

1) Convergence analysis: Here, we analyze Algorithm 1 in terms of convergence. Before we start analyzing the convergence, we should note that the values of $P_{j, i} \forall j$ after Step 3 are different from the values of $P_{j, i} \forall j$ after step 4 (i.e., the values of $P_{j, i} \forall j$ change twice in the same iteration). Specifically, in Step 3, the power values are changed because of updating the value of $v_{i}$, while in Step 4, the power values 
are changed because of updating the values of $\mu ? s$. To avoid this confusion, we denote the values of $P_{j, i} \forall j$ after Step 3 by $P_{j, i}^{(3)}$ and denote the values of $P_{j, i} \forall j$ after Step 4 by $P_{j, i}^{(4)}$.

At any $q^{\text {th }}$ iteration, it can be shown that in Step 3, the variable $v_{i}(q)$ steers the the summation of powers to be equal to $P_{i, \max }$ (i.e., to achieve Constraint (11c) with equality). In other words, if we find $v_{i}(q)$ at Step 3 and substitute it in (17) $\forall j$, we find that $\sum_{j=1}^{N_{i}} P_{j, i}^{(3)}(q)=P_{i, \max }$. On the other hand, Step 4 implies that each $P_{j, i}^{(3)}$ that is less than the corresponding $P_{j, i}^{\min }$ (i.e. each $P_{j, i}^{(3)}$ that does not satisfy the corresponding constraint in (11b)) is increased by increasing the associated $\mu_{j, i}$ to achieve that $P_{j, i}^{(4)}(q)=P_{j, i}^{\text {min }}$. This increase in power yields violating Constraint (11c) to be as $\sum_{j=1}^{N_{i}} P_{j, i}^{(4)}(q) \geq P_{i, \max }$. Hence, in the $(q+1)^{t h}$ iteration, $v_{i}(q+1)$ will be greater than $v_{i}(q)$ to have that $\sum_{j=1}^{N_{i}} P_{j, i}^{(3)}(q+1)=P_{i, \max }$ again.

Without loss of generality, we assume that in the $q^{\text {th }}$ iteration, $P_{j, i}(q)<P_{j, i}^{\text {min }}$ for $j=1, . ., k-1$ and $P_{j, i}(q) \geq P_{j, i}^{\text {min }}$ for $j=k, . ., N_{i}$. From (17), we note that the increase in $v_{i}$ leads to decreasing each $P_{j, i}^{(3)} \forall j$ with keeping the constraint $\sum_{j=1}^{N_{i}} P_{j, i}^{(3)}(q+1)=P_{i, \max }$ satisfied. Hence, we have that $P_{j, i}^{(3)}(q+1)<P_{j, i}^{(3)}(q), j=K, \ldots, N_{i}$ (because Step 4 causes no change in these powers in the iteration $q)$, and $P_{j, i}^{(3)}(q+1)>P_{j, i}^{(3)}(q), j=1, \ldots, k-1$ to keep the constraint $\sum_{j=1}^{N_{i}} P_{j, i}^{(3)}(q+1)=P_{i, \max }$ satisfied. This means that $\mu_{j, i}(q+1)$ must be increased to achieve the constraint $P_{j, i}^{(4)}(q+1)=P_{j, i}^{\min }, j=1, . ., k-1$ but with less amount than what was required in $\mu_{j, i}(q)$. Consequently, with implementing one iteration more, we have

$\mu_{j, i}(q+2)-\mu_{j, i}(q+1)<\mu_{j, i}(q+1)-\mu_{j, i}(q), j=1, . ., k-1$.

Similarly,

$$
v_{i}(q+2)-v_{i}(q+1)<v_{i}(q+1)-v_{i}(q),
$$

Therefore, as the number of iterations increases, the amount of change in $v_{i}$ and all $\mu_{j, i} \forall j$ approaches zero. Thus, Algorithm 1 is convergent.

It is also important to note that in Equation (19), $v_{i}$ is a factor of the expression $1+\mu_{j, i}$, which means that starting with any initial values for $\mu_{j, i}$ will be compensated by $v_{i}$ to have the same value of $P_{j, i}$ as Equation (17) shows. Hence, Algorithm 1 does not depend on the initial values.

2) Finding the exact interference information: Now, in the VLC APs, the problem is how to find the instantaneous interference $\left(X_{j, i}\right)$ of each user, which is difficult to be found because the term $X_{j, i}$ of user $j$ depends on all powers of the APs that are allocated for the $j^{\text {th }}$ user frequency spectrum. Therefore, we provide an iterative algorithm that solves the PA problem of all the VLC APs with finding the instantaneous interference of each user. Algorithm 2 provides the optimal power allocation for a given distributed users. It is worth stating that the RF AP performs its power allocation using Algorithm 1 and it is not included in Algorithm 2. This is because the interference of the users connected to the RF $\mathrm{AP}$ is zero. In Algorithm 2, the only step that needs to $\overline{\text { Algorithm } 2 \text { Power allocation for all the VLC APs with a }}$ given distributed users.

1) Each AP allocates the power for the users equally.

2) for $q=1: M$

3) Calculate $X_{j, i}^{(q)}$ for all users in the system.

4) Perform Algorithm 1 for all APs.

5) if $\sum_{i=1}^{N_{p}} \sum_{j=1}^{N_{i}}\left(X_{j, i}^{(q)}-X_{j, i}^{(q-1)}\right)^{2} \leq \epsilon$; break;

6) End for

exchange the information between APs is Step 3 (Calculating the interference). To calculate the interference $\left.X_{(} j, i\right)$, the AP $i$ must know the power allocated from the other APs for the BW used by User $j$. Therefore, the APs must exchange their power information to have the interference information at each user.

\section{B. Load Balancing}

As stated earlier, initially, each user is connected to its closest AP. Then, each AP performs its own power allocation problem as shown in the previous section. However, some APs will be overloaded, which may cause some users connected to these APs to receive a poor QoS. Therefore, after the PA optimization problem (Algorithm 2) is performed, the user with the poorest QoS is reconnected to either the RF AP or another adjacent VLC AP if the later can provide a better service and increase the system capacity. The users connected to the RF AP do not receive any interference so that the strongest candidate AP for the user (which needs to reconnect to another AP) to travel to is the RF AP. The approach here is to arrange the users as a queue starting from the user with the lowest QoS up to the highest quality serviced user. Then, each user in its turn tests if the RF AP can provide a better achievable data rate for it or not. If so, the user migrates to the RF AP; otherwise, the user transfers to another adjacent AP if that AP can provide a better achievable data rate for him. These procedures continue until no improvement in the system capacity can be achieved. From the PA Problem in (11), each VLC AP offers for each user its QoS denoted by $\gamma_{j, i}$, which is a function of the number of users connected to that AP as shown in (12). Hence, the maximum offered achievable data rate for user $j$ that is connected to the AP $i$ is given by

$$
\bar{R}_{j, k}=\max _{k \in \chi_{i}}\left(\gamma_{j, k}\right),
$$

where $\chi_{i}$ is the set of the APs that are very close to the AP $i$ and $N_{k}<N_{i}-1 \quad \forall k$. Since $\gamma_{j, k}$ depends on the value of $\beta$, if $N_{k}=0$, that means the AP $k$ offers all its resources for the coming user, and hence, $\beta=1$ in (23). Otherwise; if $N_{k} \geq 1, \beta$ is determined by the AP $k$. It is important to state that the transfer of a user connection changes the interference information, which enforces the system to re-implement Algorithm 2 with each transfer. This process of transfer continues only if there is an improvement in the system capacity. To prove the convergence of this approach, first, we should note that the step of sorting users is conducted at the beginning of every round of testing all users not with each user transfer. In addition, we cancel each user transfer 
TABLE I

SimUlation PARAMETERS

\begin{tabular}{lc} 
Name of the Parameters & Value of the Parameters \\
\hline Room hight & $3 \mathrm{~m}$ \\
Maximum bandwidth of VLC AP, $B_{\max }^{(v)}$ & $30 \mathrm{MHz}$ \\
Maximum bandwidth of RF AP, $B_{\max }^{(r)}$ & $30 \mathrm{MHz}$ \\
The physical area of a PD, $A_{p}$ & $0.1 \mathrm{~cm}^{2}$ \\
Half-intensity radiation angle, $\theta_{1 / 2}$ & $60^{\circ}$ \\
FoV semi-angle of PD, $\Theta$ & $90^{\circ}$ \\
Gain of optical filter, $g_{o f}$ & 1 \\
Refractive index, $n$ & 1.5 \\
Optical to electric conversion efficiency, $\rho$ & 1 \\
Transmitted power of the VLC AP, $P_{\max }^{(v)}$ & $4 \mathrm{watt}$ \\
Transmitted power of the RF AP, $P_{\max }^{(r)}$ & $2 \mathrm{watt}$ \\
Noise power spectral density of LiFi, $N_{0}^{(v)}$ & $10^{-21} A^{2} / \mathrm{Hz}$ \\
Variance of AWGN in RF AP, $N_{0}^{(r)}$ & $10^{-19} A^{2} / \mathrm{Hz}$ \\
\hline
\end{tabular}

from one AP to another that produces a degradation in the system capacity. Consequently, with each user transfer the system capacity increases, and as we know the capacity has a limit, which means that the convergence is occurred when we approach that capacity limit.

\section{Suboptimal Approach: Averaging the Interference}

The disadvantage of the above approach is its high complexity since with each user connection transfer, all the APs must perform the power allocation problem, which is highly complex. The reason behind that is the need for exact interference information to implement both the PA and LB together. In this section, we aim to simplify the problem solution by relaxing this demand. User $j$ experiences interference from each AP with a power that might be less or greater than the average power. This average power is calculated under the assumption that each AP distributes its power equally for its associated users. Therefore, the essence of the approach here is that instead of obtaining the instantaneous interference, we obtain the average interference that is calculated by averaging the power of the interference coming from all the APs. Therefore, the approximate inter-cell interference at user $j$ that is connected to the AP $i$ is given by

$$
X_{j, i}=\sum_{l=1, l \neq i}^{N_{p}} \frac{P_{l, \max }}{N_{i}} h_{j, l}^{2} .
$$

The above assumption significantly simplifies the problem as there is no need to implement the PA optimization at all the APs at each user connection transfer. In other words, we only need to perform the PA optimization problem (Algorithm 1) at only two APs (the departed from and the arrived to APs).

\section{Simulation Results}

In this section, we verify the capability of the proposed algorithms for enhancing the performance of the hybrid VLC/RF network. We show the convergence of the proposed algorithms and how they increase the system capacity. A $10 \times 10$ room area is assumed with 16 VLC APs and 1 RF AP fixed in the ceiling. The values of all parameters in the considered

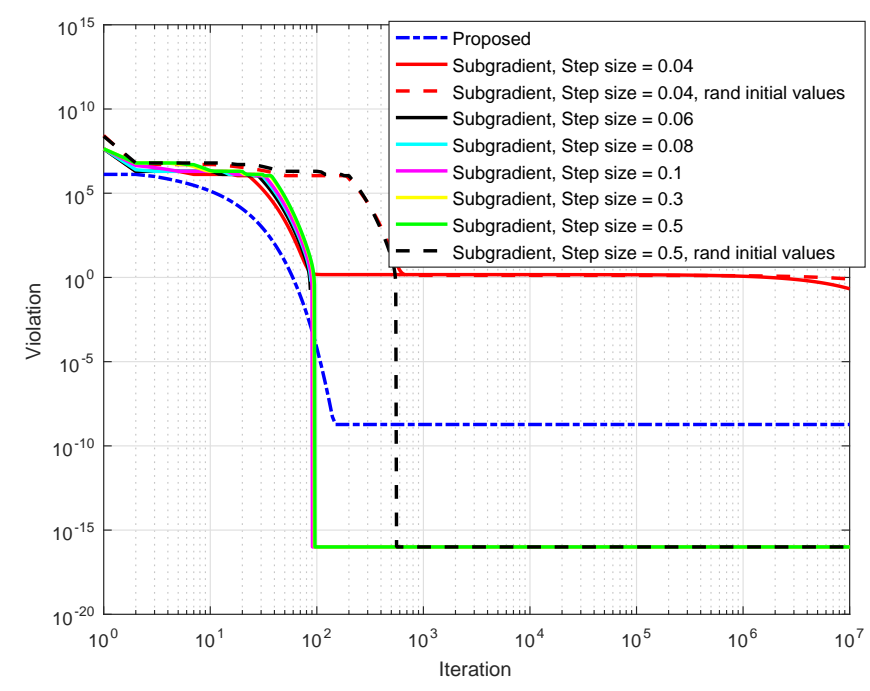

Fig. 2. Comparison between the proposed Algorithm 1 and the subgradient method with different step sizes by plotting the violation of the constraints versus number of iterations for AP $i, P_{i, \max }=10$ Watt.

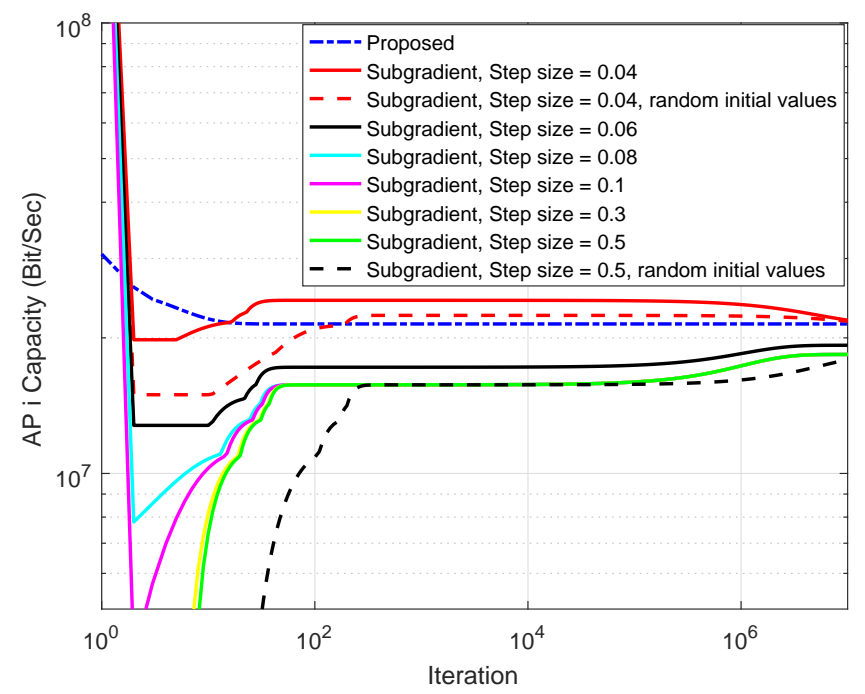

Fig. 3. Comparison between the proposed Algorithm 1 and the sub-gradient method with different step sizes by plotting the system capacity versus number of iterations, $P_{i, \max }=10$ Watt.

VLC/RF system are given in Table I. Monte-Carlo simulation is used to asses the performance of the proposed algorithms where in each simulation iteration, a uniform random number is generated between 0 and 1 . If that number is less than $\alpha$ (the probability of the availability of the LoS), the LoS component is available; otherwise, LoS component is not available.

In Fig. 2 and Fig. 3, we show the performance and convergence of the proposed Algorithm 1 implemented at AP $i$ and compare it with the subgradient method. Both figures should be analyzed together because Fig. 2 shows the maximum constraint violation of the approaches (i.e., how much the approaches are close from achieving the constraints versus the number of iterations), while Fig. 3 shows the value of the objective function for the different approaches versus the 


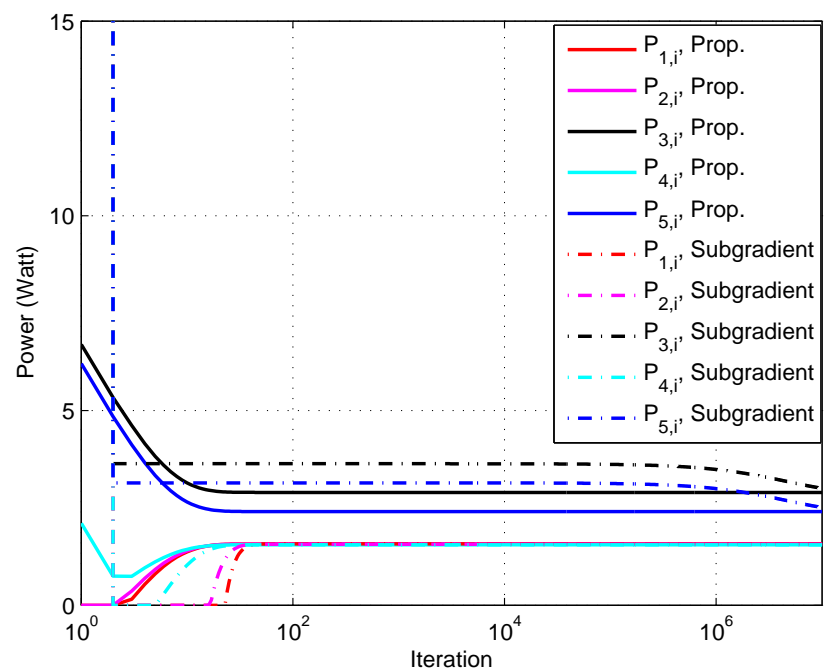

Fig. 4. Convergence of the proposed Algorithm 1 of the users powers connected to AP $i$ and compare it with the subgradient method, step size $=0.04, P_{i, \max }=10$ Watt.

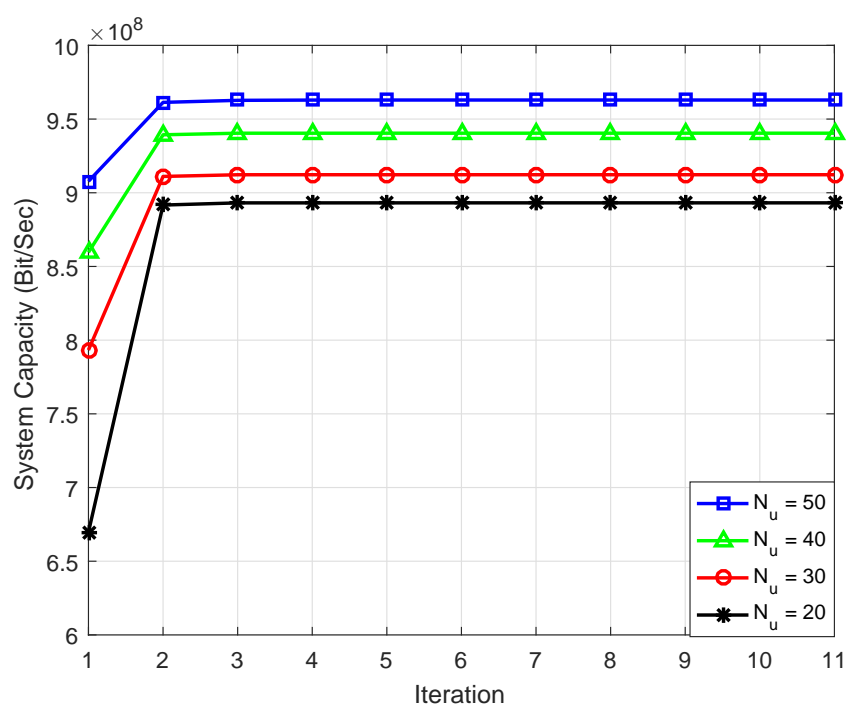

Fig. 5. System capacity versus number of iterations for Algorithm 2 with different numbers of system users.

number of iterations. It is observed that the subgradient method with large step size, such as step size $=0.5$, achieves the constraints slightly faster than the proposed approach as shown in Fig. 2, but it can not achieve the same system capacity as the proposed approach can achieve as shown in Fig. 3. In other words, if we assume that the violation tolerance is $10^{-4}$, both approaches, the proposed and the subgradient with large step size, almost satisfy the constraints after 100 iterations, while the proposed approach provides higher AP capacity than the subgradient method as shown in Fig 3. On the other hand, the subgradient method with small step size, such as step size $=0.04$, starts to satisfy the constraints after more than $10^{7}$ iterations as shown in Fig. 2, and after that huge number of iterations, it starts approaching the capacity achieved by the proposed approach as shown in Fig 3. In

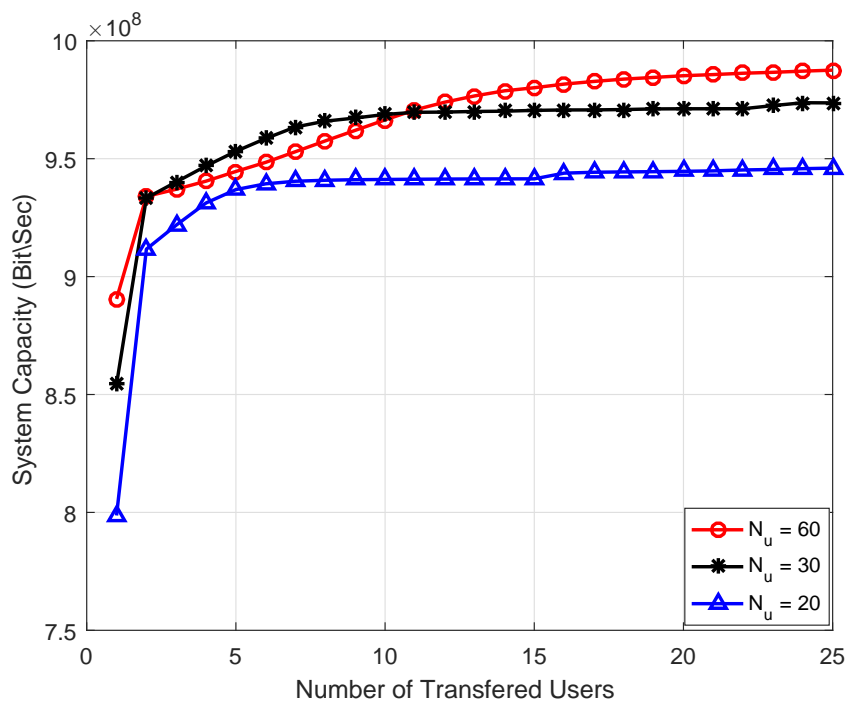

Fig. 6. System capacity versus number of transferred users for different numbers of system users.

addition, we implement the subgradient method with random initial values of the dual variables to show that the subgradient methods depends highly on the selected initial values, while the proposed algorithm does not. It is important to note that the disadvantages of the subgradient method is that the step size must be optimized to have the best performance, which is an additional problem that complicate the already subgradient method. Besides, the initial values for the dual variables must be selected carefully. It is known that in the subgradient method, the smaller step size, the closer optimal values we obtain as the number of iterations goes to infinity. Hence, as shown in Fig. 4, the proposed approach provides the same power values of the subgradient method (step size $=0.04$ ) in less than 100 iterations, while the subgradient method achieves some of those values after $10^{7}$ iterations. Fig. 4 also shows that the subgradient method violates the maximum power constraints over a wide range of iterations and it starts satisfying that constraint after huge number of iterations (10 million iterations).

Fig. 5 shows the convergence of Algorithm 2. It is clear that Algorithms 2 needs at most three iterations to converge. Furthermore, the number of iterations needed to converge does not depend on the number of users in the system, which means that the number of users does not affect the convergence of Algorithm 2. This indicates that Algorithm 2 rapidly converges to the optimal solution. In addition, it can be seen from Fig 5 that the value of the system capacity at iteration 1 is the resultant of allocating the power equally between users (Step 1 in Algorithm 2), and the value of the system capacity at iteration 2 is the result of solving the optimization problem, where the interference information is calculated from the power allocated in the first iteration which is the equal power allocation, and so on. Therefore, Fig. 5 highlights the significant contribution of the proposed allocation power algorithm over allocating the power equally among users. 


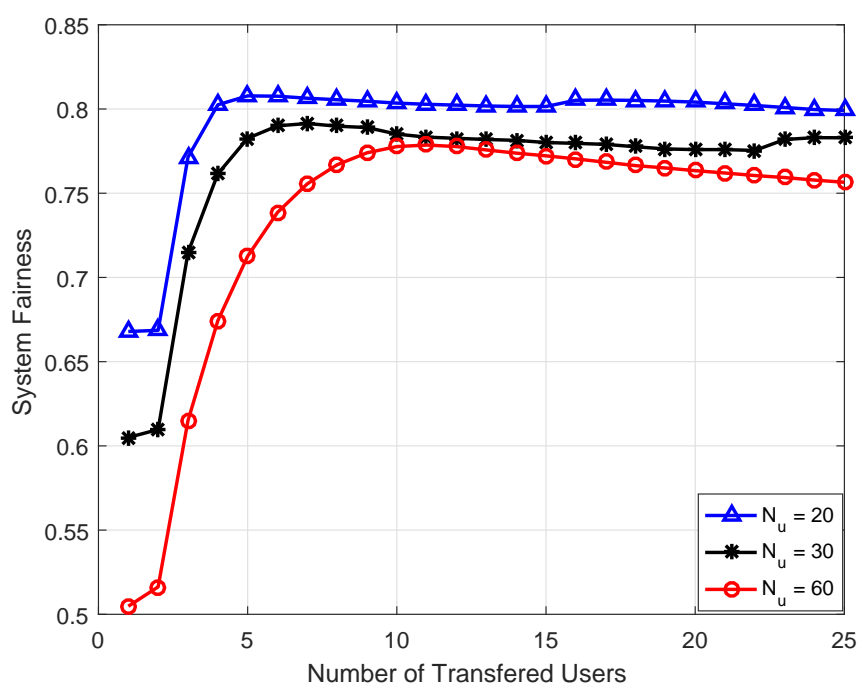

Fig. 7. System fairness versus number of transferred users for different number of system users.

In Fig. 6, the relation between the system capacity and number of reconnected users is shown with different numbers of total users. The number of transferred users means how many users transferred their connections form one AP to another. It can be seen that the more the users transfers, the better the system capacity till the system saturates. Also, it is obvious that more users transfers are needed for the system to reach the saturation point as the number of users increases, as expected. In other words, the rate of increasing the system capacity with 60 users is less than the rate of increasing the system capacity with 20 or 40 users.

The impact of total number of users on the system fairness is studied in Fig. 7. It is clear from this figure that as the number of transferred users increases, the system fairness is more enhanced with the best results achieved at the minimum number of total users. Enhancing the system fairness and capacity together with each user transfer comes from the fact that the users with poor services travel from the overloaded APs to have better services from other less overloaded APs. This helps in decreasing the variance of the received data rate among users and provides a more efficient utilization of APs resources.

Fig. 8 shows the relation between the number of users and the total system capacity for different values of $\alpha$. Here, $\alpha=1$ means no blockages is happened, while $\alpha=0.85$ means that the probability of having an objects between APs and users is 0.15 . It is shown that the difference between the performance of Algorithm 2 and the suboptimal solution (averaging the interference) is negligible. We also investigate the effect of number of users on the system fairness in Fig. 9. The approach that considers the approximate interference information slightly performs better than the approach that considers the exact interference in terms of the system fairness. This is due to the fact that the former depends on calculating interference matrix based on the assumption that the power at the APs is distributed fairly among users, which leads to a

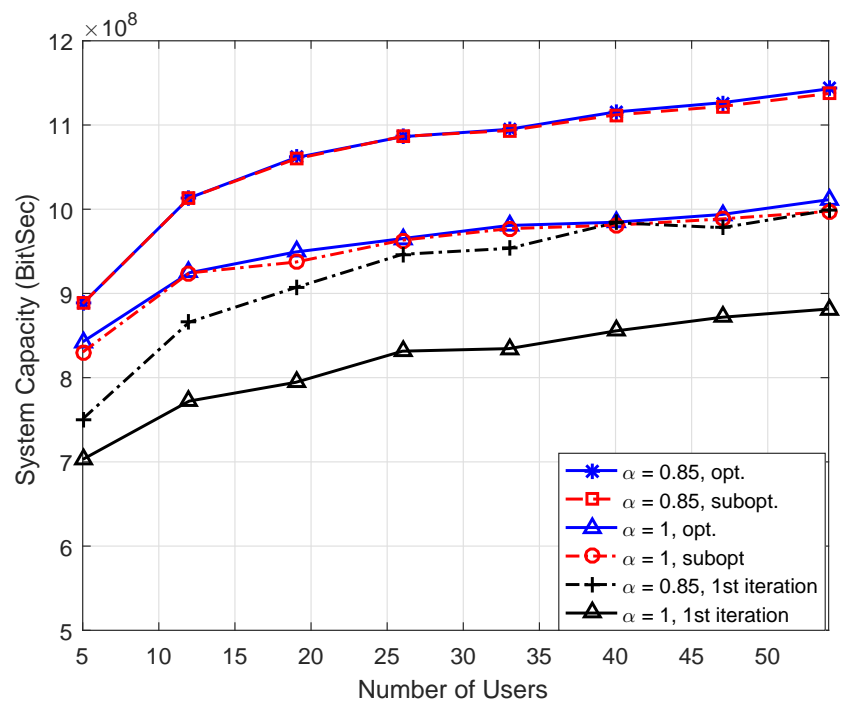

Fig. 8. Comparison between Algorithm 2 and the suboptimal approach in terms of system capacity for different values of $\alpha$.

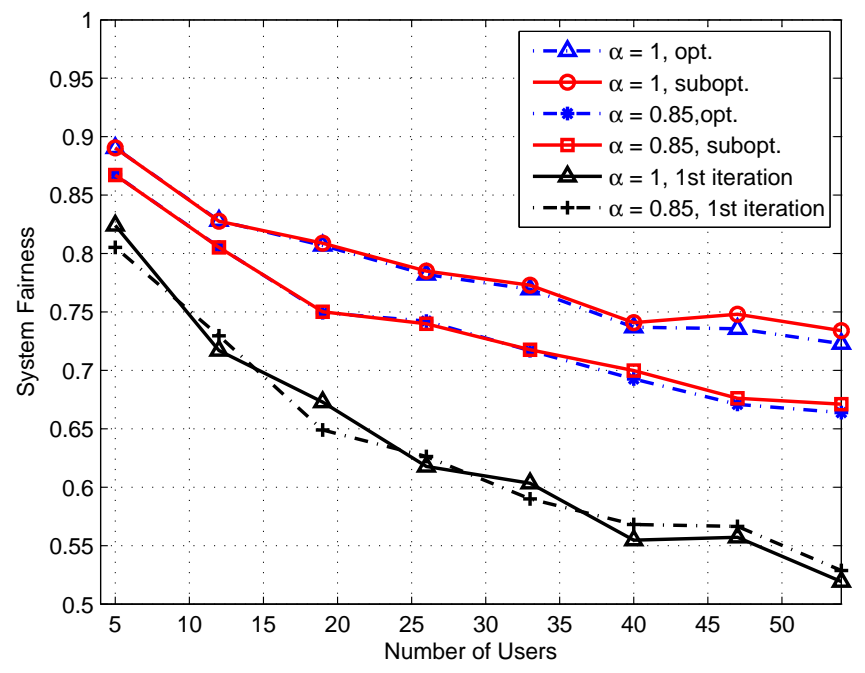

Fig. 9. Comparison between Algorithm 2 and the suboptimal approach in terms of system fairness for different values of $\alpha$.

more fair power allocation among users than the later. As can be seen from Fig. 8 and Fig. 9, as the probability of the LoS availability decreases, the system capacity increases while the fairness decreases. This is because increasing the blockages rate in the hybrid VLC/RF networks can enhance the system capacity rather than compromising it. In other words, the blockages are more likely to prevent the users from receiving the optical interference than blocking the intended signals. On the other hand, blocking some users from some VLC APs increases the variance of the received data rates among users which leads to decrease the system fairness. The figures show also how the proposed joint LB and PA algorithms significant improve the system capacity and fairness compared to the case where one iteration is implemented only (each user is assigned to its closest AP and each AP implements the PA only once). 


\section{CONCLUSION}

In this paper, a joint $\mathrm{LB}$ and PA algorithms for hybrid RF/VLC network were proposed. An iterative algorithm was proposed to maximize the total system capacity and enhance the system fairness. Two approaches were implemented: 1) the approach that is based on the exact interference information for all users, which provided better performance, 2) and a suboptimal approach that is based on the approximate interference information, which had a less complexity compared to the first approach. In the PA subproblem, we derived a new efficient algorithm that finds the optimal dual variables after formulating them in terms of each other. This new algorithm provided a faster convergence and a better performance than the traditional subgradient method. As a future work, we recommend to implement the joint power and bandwidth allocation with load balancing in mobile users.

\section{ACKNOWLEDGMENT}

This work was funded by the Deanship of Scientific Research in King Fahd University of Petroleum \& Minerals (KFUPM) through grant number KAUST004. The work was also supported by the National Plan for Science, Technology and Innovation (Maarifah) - King Abdulaziz City for Science and Technology - through the Science and Technology Unit at KFUPM - the Kingdom of Saudi Arabia, under grant number 15-ELE4157-04. The authors would like also to acknowledge the KFUPM-KAUST research initiative resulted from this research work.

\section{REFERENCES}

[1] M. Obeed, A. M. Salhab, S. A. Zummo, and M. S. Alouini, "Joint load balancing and power allocation for hybrid vlc/rf networks," in Proc. IEEE Global Commun. Conf. (Globecom), Dec 2017, pp. 1-6.

[2] C.-L. Tsai and Z.-F. Xu, "Line-of-sight visible light communications with InGaN-based resonant cavity LEDs," IEEE Photon. Technol. Lett., vol. 25, no. 18, pp. 1793-1796, 2013.

[3] Y. Wang, D. A. Basnayaka, and H. Haas, "Dynamic load balancing for hybrid Li-Fi and RF indoor networks," in Proc. IEEE Int. Conf. Commun. Workshop (ICCW), London, UK, 2015, pp. 1422-1427.

[4] M. Kashef, M. Abdallah, K. Qaraqe, H. Haas, and M. Uysal, "On the benefits of cooperation via power control in OFDM-based visible light communication systems," in Proc. IEEE Personal, Indoor, and Mobile Radio Commun. (PIMRC) Conf., Washington, DC, USA, Sept. 2014, pp. 856-860.

[5] M. B. Rahaim, A. M. Vegni, and T. D. Little, "A hybrid radio frequency and broadcast visible light communication system," in Proc. IEEE Global Commun. Wkshps. IEEE, 2011, pp. 792-796.

[6] D. A. Basnayaka and H. Haas, "Hybrid RF and VLC systems: Improving user data rate performance of VLC systems," in Proc. IEEE Veh. Technol. Conf. (VTC Spring), Glasgow, UK, May 2015, pp. 1-5.

[7] Y. Wang and H. Haas, "Dynamic load balancing with handover in hybrid Li-Fi and Wi-Fi networks," IEEE/OSA J. Lightw. Technol., vol. 33, no. 22, pp. 4671-4682, Nov. 2015.

[8] X. Wu, M. Safari, and H. Haas, "Bidirectional allocation game in visible light communications," in Proc. IEEE Veh. Technol. Conf. (VTC Spring), Montreal, Canada, 2016, pp. 1-5.

[9] M. Kashef, M. Ismail, M. Abdallah, K. A. Qaraqe, and E. Serpedin, "Energy efficient resource allocation for mixed RF/VLC heterogeneous wireless networks," IEEE J. Selec. Areas Commun., vol. 34, no. 4, pp. 883-893, 2016.

[10] X. Li, R. Zhang, and L. Hanzo, "Cooperative load balancing in hybrid visible light communications and WiFi," IEEE Trans. Commun., vol. 63, no. 4, pp. 1319-1329, 2015.

[11] R. Zhang, H. Claussen, H. Haas, and L. Hanzo, "Energy efficient visible light communications relying on amorphous cells," IEEE J. Sel. Areas Commun., vol. 34, no. 4, pp. 894-906, 2016.
[12] X. Li, Y. Huo, R. Zhang, and L. Hanzo, "User-centric visible light communications for energy-efficient scalable video streaming," IEEE Trans. Green Commun. Netw., vol. 1, no. 1, pp. 59-73, 2017.

[13] D. W. K. Ng, E. S. Lo, and R. Schober, "Energy-efficient resource allocation in multi-cell ofdma systems with limited backhaul capacity," IEEE Trans. Wirel. Commun., vol. 11, no. 10, pp. 3618-3631, 2012.

[14] H. Wang, A. Liu, and X. Pan, "Optimization of joint power and bandwidth allocation in multi-spot-beam satellite communication systems," Math. Problems in Eng., vol. 2014, 2014.

[15] J. Tang, D. K. C. So, E. Alsusa, K. A. Hamdi, A. Shojaeifard, and K.-K. Wong, "Energy-efficient heterogeneous cellular networks with spectrum underlay and overlay access," IEEE Trans. Vehicular Technol., 2017.

[16] W.-C. Pao and Y.-F. Chen, "Adaptive gradient-based methods for adaptive power allocation in ofdm-based cognitive radio networks," IEEE transa. vehicular technol, vol. 63, no. 2, pp. 836-848, 2014.

[17] S. Xiao, X. Zhou, Y. Yuan-Wu, G. Y. Li, and W. Guo, "Robust resource allocation in full-duplex-enabled ofdma femtocell networks," IEEE Trans. Wirel Commun., vol. 16, no. 10, pp. 6382-6394, 2017.

[18] J. M. Kahn and J. R. Barry, "Wireless infrared communications," Proc. IEEE, vol. 85, no. 2, pp. 265-298, 1997.

[19] W. Guo, S. Wang, and X. Chu, "Capacity expression and power allocation for arbitrary modulation and coding rates," in Proc. IEEE Wireless Commun. and Netw. Conf. (WCNC), Shanghai, China, 2013, pp. 3294-3299.

[20] S. Boyd and L. Vandenberghe, Convex Optimization. Cambridge University Press, 2004.

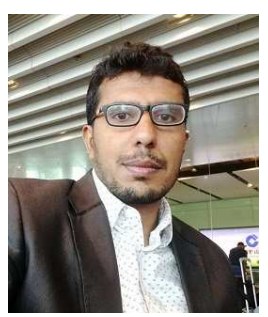

Mohanad Obeed (S'17) received the B.Eng. degree in computer and communication engineering from Taiz University, Taiz, Yemen, in 2008, the M.Sc. degree in electrical engineering from King Fahd University of Petroleum and Minerals (KFUPM), Dhahran, Saudi Arabia, in 2016

$\mathrm{He}$ is currently pursuing the Ph.D. degree at KFUPM, Dhahran, Saudi Arabia. His research interests include visible light communications, cooperative communication, resource allocation, convex optimization, physical layer security, and energy

harvesting.

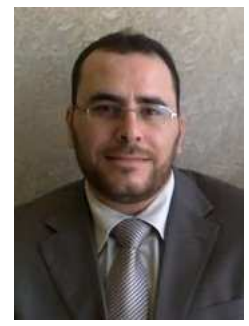

Anas M. Salhab (S'11-M'14-SM'17) received the B.Sc. degree in electrical engineering from Palestine Polytechnic University, Hebron, Palestine, in 2004, the M.Sc. degree in electrical engineering from Jordan University of Science and Technology, Irbid, Jordan, in 2007, and the Ph.D. degree from King Fahd University of Petroleum and Minerals (KFUPM), Dhahran, Saudi Arabia, in 2013. From 2013 to 2014, he was a Postdoctoral Fellow with the Electrical Engineering Department, KFUPM. He is currently an Assistant Professor and the Assistant Director of the Science and Technology Unit with the Deanship of Scientific Research, KFUPM. His research interest spans special topics in modeling and performance analysis of wireless communication systems, including cooperative relay networks, cognitive radio relay networks, free space optical networks, visible light communications, and co-channel interference. He was selected as an Exemplary Reviewer by the IEEE WIRELESS COMMUNICATIONS LETTERS for his reviewing service in 2014. Recently, he received the KFUPM Best Research Project Award as a Co-investigator among the projects in 2013/2014 and 2014/2015. 


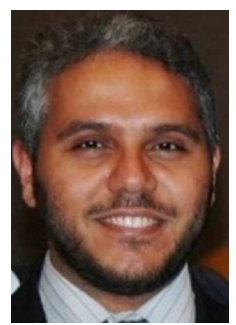

Salam A. Zummo (M'00-SM'08) received the B.Sc. and M.Sc. degrees in electrical engineering from the King Fahd University of Petroleum and Minerals (KFUPM), Dhahran, Saudi Arabia, in 1998 and 1999, respectively, and the Ph.D. degree from the University of Michigan, Ann Arbor, USA, in 2003. He is currently a Professor with the Electrical Engineering Department, KFUPM. Prof. Zummo has six issued U.S. patents and authored over 100 papers in reputable journals and conference proceedings. His research interests are in the area of wireless communications, including cooperative diversity, cognitive radio, multiuser diversity, scheduling, MIMO systems, error control coding, multihop networks, and interference modeling and analysis in wireless systems. He received the Saudi Ambassador Award for early Ph.D. completion in 2003, and the British Council/BAE Research Fellowship Awards in 2004 and 2006. He also received the KFUPM Excellence in Research Award from 2011 to 2012.

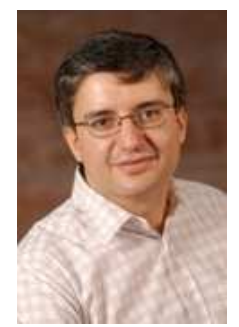

Mohamed-Slim Alouini (S'94, M'98, SM'03, F'09) was born in Tunis, Tunisia. He received the Ph.D. degree in Electrical Engineering from the California Institute of Technology (Caltech), Pasadena, CA, USA, in 1998. He served as a faculty member in the University of Minnesota, Minneapolis, MN, USA, then in the Texas A\&M University at Qatar, Education City, Doha, Qatar before joining King Abdullah University of Science and Technology (KAUST), Thuwal, Makkah Province, Saudi Arabia as a Professor of Electrical Engineering in 2009. His current research interests include the modeling, design, and performance analysis of wireless communication systems. 